\title{
Assessment of two pressure-velocity coupling strategies for local meshless numerical method
}

\author{
G. Kosec ${ }^{1,2}$, R. Vertnik ${ }^{2,3} \&$ B. Šarler ${ }^{2,4}$ \\ ${ }^{1}$ Jožef Stefan Institute, Laboratory for Parallel and Distributed \\ Computing, Slovenia \\ ${ }^{2}$ University of Nova Gorica, Laboratory for Multiphase Processes, \\ Slovenia \\ ${ }^{3}$ Štore steel, d.o.o. Research, Slovenia \\ ${ }^{4}$ Centre of Excellence for Biosensors, Automation and Process Control, \\ Laboratory for Advanced Materials Systems, Slovenia
}

\begin{abstract}
The performance of two pressure-velocity coupling strategies, associated with the primitive variable solution of incompressible Newtonian fluid with the meshless Local Radial Basis Function Collocation Method (LRBFCM), is compared with respect to computational efficiency, stability, accuracy and spatial convergence. The LRBFCM is structured with multiquadrics on five noded support domains. The explicit time stepping is used. The BackwardFacing Step problem (BFS) has been selected as a benchmark problem, previously tackled by several numerical methods. The semi-local fractional step method (FSM) and completely local pressure-velocity couplings (LPVC) are compared. The numerical results are validated against previously published data. The results are represented and compared in terms of a convergence plot of the reattachment position. We show that both approaches provide reasonable results; however, LVPC is less computationally complex and less stable in comparison to FSM.
\end{abstract}

Keywords: meshfree methods, radial basis functions, collocation, convective-diffusive problems, adaptation, refinement, melting, fluid flow, Newtonian fluids. 


\section{Introduction}

The meshless methods belong to a class of numerical methods where an arbitrarily distributed set of nodes, without any additional topological relations between them, is used. There exist several meshless methods [1-5]; however, this work is focused on one of the simplest classes of meshless methods in development today, the point interpolation Radial Basis Function Collocation Method (RBFCM) [6]. In the present paper we use a local variant of RBFCM [6], the Local Radial Basis Function Collocation Method (LRBFCM). The main advantage of the local approach is in consideration of the multivariate data fitting and calculation of spatial derivatives through a local support domain. Consequently, the computational basis is simplified, since several small systems of algebraic equations are solved instead of a global algebraic equation system. Such approach has been already successfully applied to several thermo-fluid situations, ranging from the basic diffusion problems to complex highly nonlinear and coupled technologically relevant situations [5, 7-14]. Important features of meshless methods represent straightforward application of dynamic node distribution [15], and stable behaviour on non-regular node distributions [11].

This paper is focused on application of LRBFCM in fluid mechanics, more precisely, in channel flow. In our past publications we have already demonstrated the applicability of LRBFCM in fluid flow computations. The LPVC (in the context of meshless methods) was for the first time introduced in [10] and further investigated regarding more complex physical systems in $[12,16,17]$, where only closed and impermeable domains have been considered. The open domain problems were dealt with (in the context of meshless methods) the FSM approach in [14, 18].

In this paper we compare the performance of LPVC and FSM in open domain problems, which seems to appear more demanding than the closed ones. The well-known Backward-Facing Step problem [19-25] (BFS) is considered since its solution is well coped with in the literature, and is at the same time complex enough to challenge the proposed numerical techniques.

The principal message of the present paper is a quantitative comparison of two meshless based numerical procedures that differ only in pressure-velocity coupling strategies. The Chorin's FSM [21] is semi-global, and as such requires solution of a global system of algebraic equations for pressure, while LPVC is completely local. Both strategies show reasonably good convergence behavior as well as good agreement with already published data [22].

\section{Problem definition}

The BFS problem of the laminar incompressible Newtonian fluid flow over a backward-facing step in two dimensions is considered. It represents a standard test for investigating the flow separation and reattachment. It has been already used by numerous researchers as a benchmark test for various numerical methods [19-25]. The domain of the problem is characterized by a planar 
channel with sudden change of the geometry (Figure 1), which governs the flow separation, and generation of several re-circulating zones downward the step, described by positions $\left(p_{x 1}, p_{x 2}, p_{x 3}\right)$. The flow is modelled by the Navier Stokes equations

$$
\begin{gathered}
\nabla \cdot \mathbf{v}=0, \\
\rho \frac{\partial \mathbf{v}}{\partial t}+\rho \nabla \cdot(\mathbf{v v})=-\nabla P+\nabla \cdot(\mu \nabla \mathbf{v}),
\end{gathered}
$$

with $t, \mathbf{v}\left(v_{x}, v_{y}\right), P, \mu$ and $\rho$ standing for time, velocity, pressure, viscosity and density, respectively. The Cartesian coordinates are used, and the position vector is defined as $\mathbf{p}=\left(p_{x}, p_{y}\right)$. The rectangular domain is normalized to height $2 h=1$. Beginning of the computational domain is considered at the location of the step, where the flow enters the channel. The step height is set to $h$. The channel length is set to $30 \mathrm{~h}$. The flow dynamics is characterized by the Reynolds number (Re), defined as

$$
\operatorname{Re}=\frac{\rho v_{0} 2 h}{\mu},
$$

with $v_{0}$ standing for the amplitude of the inlet velocity. At the inlet, the fullydeveloped velocity profile is set [22]

$$
v_{x}\left(p_{y}\right)=v_{0} p_{y}\left(0.5-p_{y}\right) ; 0.0 \leq p_{y} \leq 0.5 .
$$

At the outlet boundary, the Neumann boundary conditions for velocity components are prescribed and are all set equal to zero. At the walls, nonpermeable and no-slip conditions are used. The schematics of the computational test are represented in Figure 1.

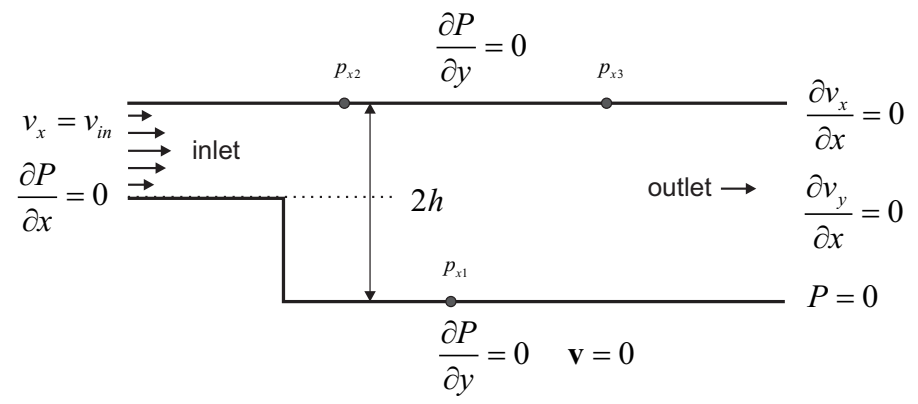

Figure 1: Problem schematics of the backward-facing step. 


\section{Solution procedure}

A general idea in the local meshless methods is the use of local sub clusters of domain nodes, named local support domains, for the approximation of the fields. Complementary to the selected support domain, an approximation function is introduced as a sum of weighted basis functions

$$
\theta(\mathbf{p})=\sum_{n=1}^{N_{\text {Basis }}} \alpha_{n} \Psi_{n}(\mathbf{p})
$$

where $\theta, N_{\text {Basis }}, \alpha_{n}$ and $\Psi_{n}$ stand for the approximation function, the number of basis functions, the approximation coefficients, and the basis functions, respectively. The basis could be selected arbitrarily (monomials, radial basis functions, ...). In this paper, Hardy's Multiquadrics (MQs),

$$
\Psi_{n}(\mathbf{p})=\sqrt{\left(\mathbf{p}-\mathbf{p}^{n}\right) \cdot\left(\mathbf{p}-\mathbf{p}^{n}\right) / \sigma_{C}^{2}+1},
$$

with $\sigma_{C}$ standing for the free shape parameter of the basis function, are used. MQ's are selected. By taking into account all support domain nodes and equation (5) the approximation system is obtained. In this paper we use collocation (the number of support nodes is the same as the number of the basis functions). An arbitrary spatial differential operation $L$ can be applied on the approximation function in the following way

$$
L \theta(\mathbf{p})=\sum_{n=1}^{N_{\text {Basis }}} \alpha_{n} L \Psi_{n}(\mathbf{p})
$$

In general, the system (5) has to be solved only when the support domain topology changes and therefore the computation can be optimized by computing $\Psi^{-1}$ in a pre-process. Furthermore, the computation of the coefficients and the evaluation of differential operators can be combined. All information about the numerical approach and the local nodal topology can be stored in a predefined vector, which has to be re-evaluated only when the topology of the nodes changes. The differential operator vector $\chi_{m}^{L}$ is introduced as

$$
\chi_{m}^{L}(\mathbf{p})=\sum_{n=1}^{N} \Psi_{n m}^{-1} L\left(\Psi_{n}(\mathbf{p})\right) .
$$

The introduced formalism holds in general and therefore the general notation for partial differential operator $(L)$ is used. However, in the present work, only operators $\nabla$ and $\nabla^{2}$ are employed

$$
\chi_{m}^{\nabla^{2}}(\mathbf{p})=\sum_{n=1}^{N} \Psi_{n m}^{-1} \sum_{\varepsilon} \frac{\partial^{2}}{\partial p_{\varepsilon}^{2}} \Psi_{n}(\mathbf{p}),
$$




$$
\chi_{m}^{\partial / \partial p_{\varepsilon}}(\mathbf{p})=\sum_{n=1}^{N} \Psi_{n m}^{-1} \frac{\partial}{\partial p_{\varepsilon}} \Psi_{n}(\mathbf{p}) .
$$

The structured formulation is convenient since most of the complex and CPU demanding operations are performed in the pre-process phase. For all inner temporal loop operations only $N$ floating point operations (FLOPS) are needed for evaluation of an arbitrary partial differential operator. The implementation of the Dirichlet boundary condition is straightforward. In order to implement Neumann and Robin boundary conditions, special interpolation is needed. In the present numerical framework, the computation of Neumann and Robin boundary conditions can be simplified through the use of the differential operator vector. Consider Neumann boundary condition

$$
\begin{gathered}
a \frac{\partial \theta}{\partial \mathbf{n}}+b \theta=\theta_{B C}, \\
\theta_{0}=\frac{\theta_{B C}-a \sum_{m=2}^{N_{\text {Suh }}} \chi_{m}^{\partial / \partial \mathbf{n}} \theta_{m}}{a \chi_{0}^{\partial / \partial \mathbf{n}}+b},
\end{gathered}
$$

where $\theta_{0}$ stands for the boundary node. Equation (12) simplifies to Neumann boundary condition computation if $b$ is set to zero. Such approach makes the Neumann and the Robin boundary condition computation straightforward and CPU effective. Again, only $N$ flops are needed to evaluate it, without any kind of special computational treatment on or near the boundaries.

\subsection{Local pressure-velocity coupling}

A two-level explicit time stepping is used, thus equation (2) is discretized as

$$
\hat{\mathbf{v}}=\mathbf{v}_{0}+\frac{\Delta t}{\rho}\left(-\nabla P_{0}+\nabla \cdot\left(\mu \nabla \mathbf{v}_{0}\right)+\mathbf{b}_{0}-\rho \nabla \cdot\left(\mathbf{v}_{0} \mathbf{v}_{0}\right)\right),
$$

with $\Delta t$ standing for time step, index zero marks the initial time step values and $\hat{\mathbf{v}}$ stands for intermediate velocity. Equation (13) does not take into account the mass continuity and therefore the pressure and the velocity corrections are added

$$
\hat{\mathbf{v}}^{m+1}=\hat{\mathbf{v}}^{m}+\widehat{\mathbf{v}}, \quad \hat{P}^{m+1}=\hat{P}^{m}+\widehat{P},
$$

where $m, \widehat{v}$ and $\widehat{P}$ stand for pressure velocity iteration index, velocity correction, and pressure correction, respectively. By combining the momentum and the mass continuity equations, the pressure correction Poisson equation emerges

$$
\nabla \hat{\mathbf{v}}^{m}=\frac{\Delta t}{\rho} \nabla^{2} \widehat{P}
$$


Instead of solving the global Poisson equation problem, the pressure correction is directly related to the intermediate velocity divergence

$$
\widehat{P}=\ell^{2} \frac{\rho}{\Delta t} \nabla \cdot \hat{\mathbf{v}}^{m}
$$

The proposed assumption enables direct solving of the pressure velocity coupling iteration. It is thus very fast due to only one spatial discretization operation needed in each node to evaluate the new iteration pressure and the velocity corrections. With the computed pressure correction, the pressure and the velocity can be corrected as

$$
\hat{\mathbf{v}}^{m+1}=\hat{\mathbf{v}}^{m}-\zeta \frac{\Delta t}{\rho} \nabla \widehat{P}, \quad \hat{P}^{m+1}=\hat{P}^{m}+\zeta \widehat{P},
$$

where $\zeta$ stands for the relaxation parameter. The iteration is performed until the criterion $\nabla \cdot \hat{\mathbf{v}}<\varepsilon_{V}$ is met in all computational nodes. After successful pressurevelocity iteration, the algorithm continues with the next time step. The LPVC approach is similar to the artificial compressibility method (ACM), which has been recently under intense research in connection with Finite Volume Method $[26,27]$ and in connection with Finite Element Method [28]. A similar approach, in the framework of the FDM, is SOLA algorithm [29]. However, the proposed LPVC approach retains the correct time transient which is, in general, not the case in SOLA and ACM approaches.

\subsection{Fractional step approach}

The pressure is omitted from temporally discretized form of momentum equation in FSM approach

$$
\hat{\mathbf{v}}=\mathbf{v}_{0}+\frac{\Delta t}{\rho}\left(\nabla \cdot\left(\mu \nabla \mathbf{v}_{0}\right)+\mathbf{b}_{0}-\rho \nabla \cdot\left(\mathbf{v}_{0} \mathbf{v}_{0}\right)\right) .
$$

Again, the equation (18) does not take into account the mass continuity. In a similar manner as in 3.1 , the pressure Poisson equation is constructed.

$$
\nabla^{2} P=\frac{\Delta t}{\rho} \nabla \hat{\mathbf{v}} .
$$

The equation (19) is directly solved in the FSM approach. However, the locality of spatial discretization simplifies the computations to solution of a sparse system of algebraic equations. With the solved pressure, the intermediate velocity is corrected as

$$
\mathbf{v}=\hat{\mathbf{v}}-\frac{\Delta t}{\rho} \nabla P
$$


In FSM, the internal iterations are not performed. The computed pressure and corrected velocity are therefore new values and simulation proceeds to the next time step. FSM does not provide a correct time transient in general.

\section{Results}

The results are presented in terms of velocity field plot and velocity, pressure and shear stress cross section profiles for $\operatorname{Re}=800$ case. The convergence is monitored at the reattachment position $p_{x 1}$ with respect to the number of computational nodes, ranging from $150 \times 40$ to $325 \times 110$. The reattachment position is defined as a point where the wall shear stress equals zero

$$
\tau=\mu \frac{\partial v_{x}}{\partial p_{y}}=0
$$

Steady state velocity field is represented in Figure 2. The $\mathrm{x}$ axis is on the plot limited to 10 (for the sake of better visibility), although the computations are performed on domain with length 15 .

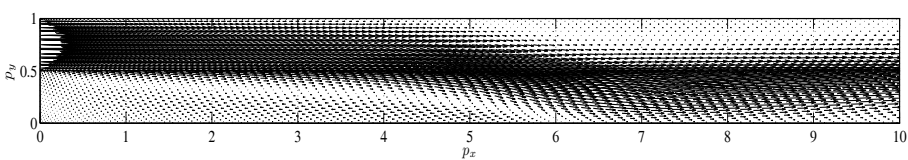

Figure 2: Problem schematics of the backward-facing step.

All computations were performed with time step $\Delta t=10^{-3}$, relaxation parameter $\zeta=10^{-7}$ and $\mathrm{MQ}$ shape parameter $\sigma_{C}=32$, normalized to the maximum distance between the nodes within the support domain.

A comparison of the results is done on velocity cross-section profiles with the already published data [22], presented as "Gartling" in Figures. Two different cross-sections are examined, at $p_{x}=7$ and $p_{x}=15$ (Figure 3). In Figure 4, the shear stress profiles at the bottom and the top walls are represented. A good agreement is achieved in all analyses. Finally, the convergence plot for both meshless based solutions is represented in Figure 5. Both solution procedures converge at the same rate with minimal differences. However, it can be seen that LPVC develops minor instabilities in the convergence plot. The behaviour is to be investigated in our future work. Our preliminary analyses show that the instabilities can be mitigated by usage of an additional stability term in pressure correction. 

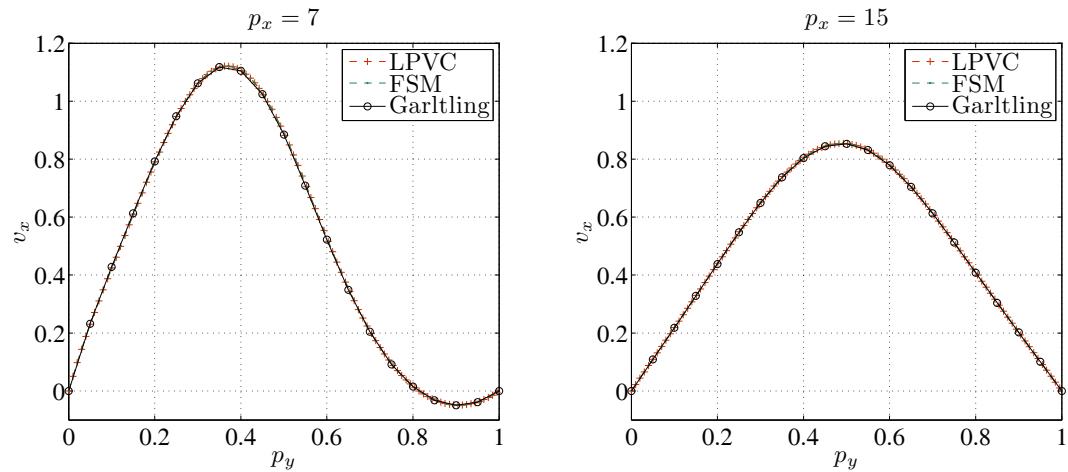

Figure 3: Comparison of vertical velocity profiles at $p_{x}=7$ and $p_{x}=15$.
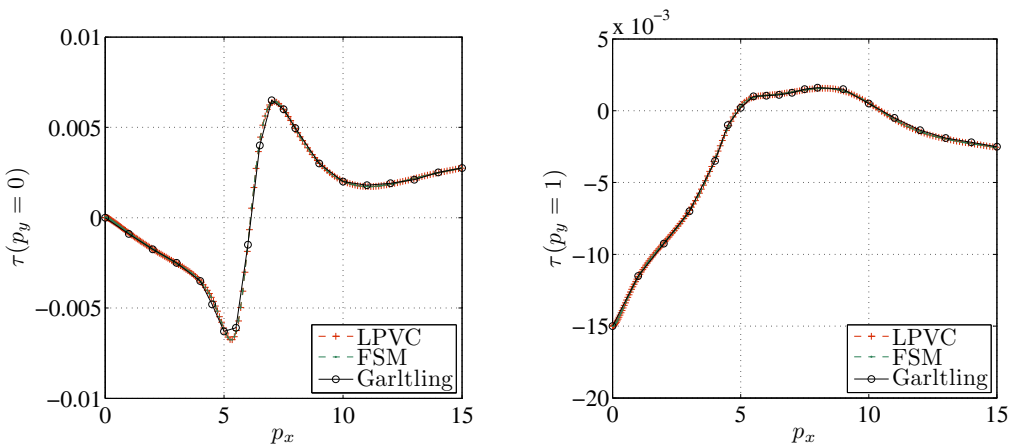

Figure 4: Comparison of shear stress profiles at the top and at the bottom wall.

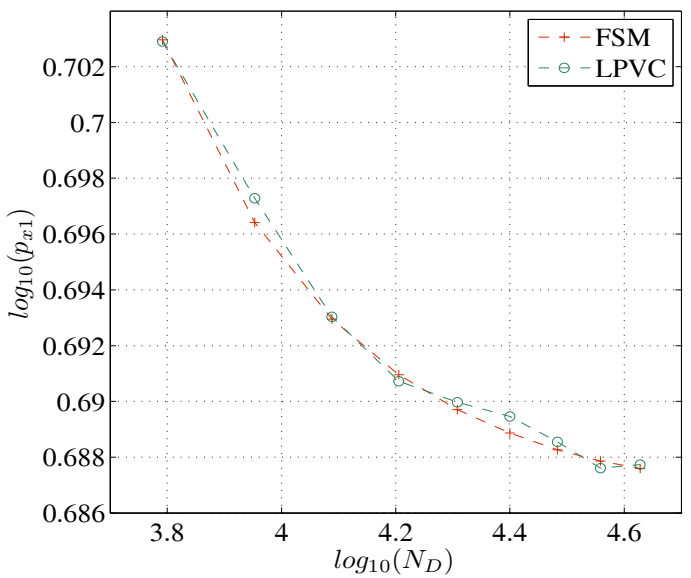

Figure 5: Convergence plot for reattachment position $p_{x 1}$ with respect to the number of the nodes $N_{D}$. 


\section{Conclusions}

Two different strategies for pressure-velocity coupling in meshless solution of laminar, incompressible Newtonian channel flow are presented. Both strategies provide good results. Comparison with published data shows good accuracy of both presented methods. The semi-local FSM behaves more stable as the LPVC. The main advantages of the LPVC approach are: ease of implementation, straightforward parallelization, and low computational cost. On the other hand, the global FSM pressure-velocity strategy does not require a consideration of any extra relaxation parameter, at the cost of higher computational complexity. The FSM approach also shows more stable convergence behavior. The main drawback of FSM as compared to the LPVC is global solution of pressure equation. First, the global system has to be constructed. This is not problematic as long as the static node distribution is used, as it can be done in preprocess. The solution of the system remains the task to be done in each time step, however. With proper iterative algorithms and pre-conditioning this overhead can be minimized. Still, the complexity is proportional to $O\left(N_{D}\right)$, while for LPVC this step is omitted. Besides a higher computational complexity, the important factor is ease of parallelization since the multicore computers are common today. The LPVC is almost ideally parallelizable as it is completely local. There is minimal communication with other parts of the computational domain. For the shared memory systems the OpenMP parallelization is trivial and extremely effective [30]. At the cost of computational complexity, however, the FSM offers more stable numerical scheme. The FSM also does not provide a proper time transient. These conclusions are preliminary and will be further investigated and supported with more analyses in the archival version of the present paper, that will be submitted to EABE.

\section{Acknowledgements}

The research was funded through Slovenian state research projects J2-4120, P2-0379 and P2-0095. The Centre of Excellence for Biosensors, Instrumentation and Process Control is an operation financed by the European Union, European Regional Development Fund and Republic of Slovenia, Ministry of Higher Education, Science and Technology. The financial support is kindly acknowledged.

\section{References}

[1] Kansa, E. J., Multiquadrics - a scattered data approximation scheme with application to computational fluid dynamics, part I. Computers and Mathematics with Applications, 19, pp. 127-145, 1990.

[2] Chen, W., New RBF collocation schemes and kernel RBFs with applications. Lecture Notes in Computational Science and Engineering, 26, pp. 75-86, 2002. 
[3] Liu, G.R., Mesh Free Methods, CRC Press: Boca Raton, 2003.

[4] Trobec, R., Šterk, M. and Robič, B., Computational complexity and parallelization of the meshless local Petrov-Galerkin method. Computers \& Structures, 87, pp. 81-90, 2009.

[5] Šterk, M. and Trobec, R., Meshless solution of a diffusion equation with parameter optimization and error analysis. Engineering Analysis with Boundary Elements, 32, pp. 567-577, 2008.

[6] Šarler, B., From global to local radial basis function collocation method for transport phenomena. Advances in Meshfree Techniques, pp. 257-282, 2007.

[7] Šarler, B. and Vertnik, R., Meshfree explicit local radial basis function collocation method for diffusion problems. Computers and Mathematics with Applications, 51, pp. 1269-1282, 2006.

[8] Vertnik, R. and Šarler, B., Meshless local radial basis function collocation method for convective-diffusive solid-liquid phase change problems. International Journal of Numerical Methods for Heat and Fluid Flow, 16, pp. 617-640, 2006.

[9] Divo, E. and Kassab, A. J., Localized meshless modeling of naturalconvective viscous flows. Numerical Heat Transfer, B129, pp. 486-509, 2007.

[10] Kosec, G. and Šarler, B., Solution of thermo-fluid problems by collocation with local pressure correction. International Journal of Numerical Methods for Heat and Fluid Flow, 18, pp. 868-882, 2008.

[11] Trobec, R., Kosec, G., Sterk, M. and Sarler, B., Comparison of local weak and strong form meshless methods for 2-D diffusion equation. Engineering Analysis with Boundary Elements, 36, pp. 310-321, 2012.

[12] Kosec, G., Založnik, M., Šarler, B. and Combeau, H., A Meshless Approach Towards Solution of Macrosegregation Phenomena. Computers, materials \& continua, 580, pp. 1-27, 2011.

[13] Vertnik, R., Založnik, M. and Šarler, B., Solution of transient direct-chill aluminium billet casting problem with simultaneous material and interphase moving boundaries by a meshless method. Engineering Analysis with Boundary Elements, 30, pp. 847-855, 2006.

[14] Vertnik, R. and Šarler, B., Local collocation approach for solving turbulent combined forced and natural convection problems. Advances in Applied Mathematics and Mechanics, 3, pp. 259-279, 2011.

[15] Kosec, G. and Šarler, B., H-Adaptive Local Radial Basis Function Collocation Meshless Method. CMC: Computers, Materials \& Continua, 26, pp. 227-254, 2011.

[16] Kosec, G. and Šarler, B., Local RBF collocation method for Darcy flow. CMES: Computer Modeling in Engineering \& Sciences, 25, pp. 197-208, 2008.

[17] Kosec, G. and Šarler, B., Solution of phase change problems by collocation with local pressure correction. CMES: Computer Modeling in Engineering \& Sciences, 47, pp. 191-216, 2009. 
[18] Vertnik, R. and Šarler, B., Solution of incompressible turbulent flow by a mesh-free method. CMES: Computer Modeling in Engineering \& Sciences, 44, pp. 66-95, 2009.

[19] Armaly, B. F., Durst, F., Pereira, J. C. F. and Schönung, B., Experimental and theoretical investigation of backward-facing step flow. Journal of Fluid Mechanics, 127, pp. 473-496, 1983.

[20] Chiang, T. P. and Sheu, T. W. H., A numerical revisit of backward-facing step flow problem. Physics of fluids, 11, pp. 862, 1999.

[21] Chorin, A.J., A numerical method for solving incompressible viscous flow problems. Journal of Computational Physics, 2, pp. 12-26, 1967.

[22] Gartling, D. K., A test problem for outflow boundary conditions-flow over a backward-facing step. International Journal for Numerical Methods in Fluids, 11, pp. 953-967, 1990.

[23] Gresho, P. M., Gartling, D. K., Torczynski, J. R., Cliffe, K. A., Winters, K. H., Garratt, T. J., Spence, A. and Goodrich, J. W., Is the steady viscous incompressible two-dimensional flow over a backward-facing step at $\mathrm{Re}=$ 800 stable? International Journal for Numerical Methods in Fluids, 17, pp. 501-541, 1993.

[24] Ramšak, M. and Škerget, L., A subdomain boundary element method for high-Reynolds laminar flow using stream function-vorticity formulation. International Journal for Numerical Methods in Fluids, 46, pp. 815-847, 2004.

[25] Zhang, X. H, Ouyang, J. and Zhang, L., Characteristic based split (CBS) meshfree method modeling for viscoelastic flow. Engineering Analysis with Boundary Elements, 34, pp. 163-172, 2010.

[26] Rahman, M. M. and Siikonen, T., An artificial compressibility method for viscous incompressible and low Mach number flows. Internation Journal of Numerical Methods in Engineering, 75, pp. 1320-1340, 2008.

[27] Malan, A. G. and Lewis, R. W., An artificial compressibility CBS method for modelling heat transfer and fluid flow in heterogeneous porous materials. International Journal for Numerical Methods in Engineering, 87, pp. 412-423, 2011.

[28] Traivivatana, S., Boonmarlert, P., Thee, P., Phongthanapanich, S. and Dechaumphai, P., Combined adaptive meshing technique and characteristic-based split algorithm for viscous incompressible flow analysis. Applied Mathematics and Mechanics, 28, pp. 1163-1172, 2007.

[29] Hong, C. P., Computer Modelling of Heat and Fluid Flow Materials Processing, Institute of Physics Publishing Bristol, 2004.

[30] Kosec, G., Trobec, R., Depolli, M. and Rashkovska, A.: Multicore parallelization of a meshless PDE solver with OpenMP, ParNum 11, 2011. 